\title{
Eficacia de la terapia manual articulatoria vertebral en pacientes con migraña
}

\author{
Elena Muñoz Gómez \\ Universitat de València (España)
}

\begin{abstract}
La migraña es uno de los trastornos del sistema nervioso más comunes y se considera la tercera causa de incapacidad en el mundo en menores de 50 años. El tratamiento de la migraña mediante terapia manual articulatoria vertebral (TMAV) puede ser efectivo, sin embargo, existe más controversia que en otros tipos de cefaleas. El objetivo del presente estudio es valorar los cambios producidos tras la aplicación de TMAV en pacientes con migraña en cuanto a la intensidad del dolor y su impacto.44 participantes con una edad media de 39 años (desviación típica $=9.47$ ) con migraña, fueron divididos en dos grupos de forma aleatoria: 1) grupo TMAV $(n=22)$, 2) grupo control $(n=22)$. Se realizaron dos evaluaciones, pretratamiento y postratamiento, y se emplearon la Escala Visual Analógica (EVA) y el Cuestionario de Impacto de las Cefaleas (HIT-6). No hubo diferencias significativas entre los dos factores (es decir, medidas tiempo y grupos) ni en EVA ni en HIT-6 ( $p>0.05)$, sin embargo, hubo diferencias significativas en el grupo TMAV entre el pretratamiento y el postratamiento en EVA $(p=0.004)$ y en HIT-6 $(p=0.014)$. La TMAV en pacientes con migraña, disminuye la intensidad del dolor y el impacto asociado a la migraña, y podría ser un tratamiento adecuado en combinación con la medicación.
\end{abstract}

Palabras clave: Migraña, cefalea, fisioterapia, terapia manual.

Efficacy of manual articular vertebral therapy in patients with migraine. Migraine is one of the most common nervous system disorders and it is the third cause of disability in under 50s. Migraine treatment using manual vertebral articulatory therapy (MVAT) may be effective, however, there ir more controversy than in other types of headaches. The objective of this study is to assess the changes produced after the application of MVAT in patients with migraine in terms of pain intensity and its impact. 44 participants with a mean age of 39 years (standard deviation= 9.47) with migraine, were divided into two groups randomly: 1) MVAT group ( $n=22), 2)$ control group $(n=22)$. Two evaluations were carried out, pretreatment and post-treatment, and the Visual Analog Scale (VAS) and the Headache Impact Questionnaire (HIT-6) were used. There were no significant differences between the two factors (i.e. time and group measures) in either VAS or HIT6 ( $p>0.05$ ), however, there were significant differences in the MVAT group between pretreatment and post-treatment. post-treatment in VAS $(p=0.004)$ and in HIT-6 $(p=0.014)$. MVAT in patients with migraine, decreases the intensity of pain and the impact associated with migraine, and could be an appropriate treatment in combination with medication.

Keywords: Migraine, headache, physiotherapy, manual therapy.

Correspondencia: Elena Muñoz Gómez. Facultat de Fisioteràpia.. Universitat de València.. C/ Gascó Oliag, 5. C.P.: 46010 Valencia (España). E-mail: Elena.MunozGomez@uv.es 
Las cefaleas primarias son uno de los trastornos del sistema nervioso más comunes (OMS, 2019). La Sociedad Internacional de Cefaleas (Headache Classification Committee of the International Headache Society (IHS) (2013), clasifica la migraña como cefalea primaria, de localización unilateral, pulsátil, de intensidad moderada o severa, agravada por la actividad física rutinaria, se acompaña de náusea y/o fotofobia y fonofobia y es precedida o agravada por aura en algunos pacientes (IHS, 2013). La migraña se considera la tercera causa de incapacidad en el mundo en menores de 50 años (Steiner, Stovner, y Vos, 2016), y tiene un alto impacto en la calidad de vida (Abu Bakar et al., 2016).

Recientes estudios describen la migraña como un trastorno complejo y variable del sistema nervioso, influenciado por la genética y el ambiente (Goadsby et al., 2017). La asociación entre 38 genomic loci y la migraña ha sido validada en diversos estudios, pero la gran diversidad de estos genes y la probabilidad de que, en muchos casos, la migraña implica una interacción entre múltiples genes y factores epigenéticos, subraya la complejidad del trastorno (Charles, 2018).

El tratamiento por excelencia de la migraña es el farmacológico, pero el tratamiento no farmacológico más utilizado en el manejo de las cefaleas primarias es la terapia manual (TM), con el objetivo de aliviar el dolor (Moore, Sibbritt, y Adams, 2017).

La zona cervical y torácica de los pacientes con migraña presentan disfunciones músculo-esqueléticas (Luedtke, Starke, y May, 2018), lo cual está relacionado con la activación del sistema trigeminal y los síntomas de la migraña (Ferracini et al., 2017). Por tanto, la TM, incluyendo manipulación o movilización articular espinal, intervenciones de tejido blando, ejercicio terapéutico y terapias con punción, ha sido estudiada en pacientes con cefaleas primarias, y ofrecen posibles efectos positivos (Bevilaqua-Grossi et al., 2016; Calixtre et al., 2018; Cerritelli et al., 2015; Chaibi et al., 2017; Chaibi, Benth, Tuchin, y Russell, 2017; Dunning et al., 2016; Espí-López et al., 2018; Fernández-de-Las-Peñas y Cuadrado, 2016; Garrigós-Pedrón et al., 2018; Garrigós-Pedrón et al., 2018; Tuchin, Pollard, y Bonello, 2000; Voigt et al., 2011).

La terapia manual articulatoria vertebral (TMAV) puede ser efectiva en pacientes con migraña al reducir el número de episodios y la intensidad del dolor (Rist et al., 2019), sin embargo, existe más controversia que en otros tipos de cefaleas (Fernández-de-Las-Peñas y Cuadrado, 2016), y por tanto, este estudio pretende ampliar la información en esta línea.

La movilización manual y/o tratamiento manipulativo espinal es seguro y efectivo en el tratamiento con pocos y transitorios efectos adversos (Chaibi y Russell, 2019). 
El objetivo principal de este estudio es valorar los cambios producidos tras la aplicación de TMAV en pacientes con migraña en cuanto a la intensidad del dolor y su impacto.

\section{MÉTODO}

\section{Participantes}

La muestra la formaron 44 participantes (36 mujeres y 8 hombres) con una edad media de 39 años (desviación típica=9.47) con migraña con mínimo un episodio a la semana y más de un año de evolución de la cefalea. Los sujetos fueron reclutados en centros de atención primaria y diagnosticados según los criterios de IHS (2013). Los criterios de exclusión fueron estar en proceso de adaptación farmacológica las 4 semanas anteriores, tener problemas de compromiso de la arterial vertebral, radiculopatía espinal, vértigos o embarazo.

El ensayo se realizó en la Univeristat de València y fue aprobado por el Comité de Ética de la Universidad con el número de protocolo H1509655117217. Previo a la recolección de datos, los sujetos firmaron el consentimiento informado, y todos los procedimientos se realizaron bajo la Declaración de Helsinki y su actualización en 2013. Este estudio fue registrado en www.clinicaltrials.gov (NCT03555214).

\section{Diseño del estudio}

Se realizó un estudio comparativo de dos grupos, aleatorizado, prospectivo y simple ciego, en los meses de noviembre 2018 a febrero 2019. Previo a la intervención se dividió la muestra en dos grupos de forma aleatoria por un asistente externo, utilizando una secuencia de aleatorización generada por un ordenador. Se dividió en: a) grupo experimental $(n=22)$ que recibió TMAV; b) el grupo control placebo $(n=22)$. Ambos tratamientos tuvieron una duración de 4 semanas que incluyó 4 sesiones (una por semana). Las evaluaciones se realizaron al inicio y al final de la intervención.

\section{Intervención}

Grupo 1 experimental $(G E)$. Se aplicó un tratamiento con técnicas articulatorias sobre la articulación sacroilíaca y lumbosacra, vértebras dorsales altas, C7$\mathrm{D} 1$, vértebras cervicales y C0-C1-C2. Se han empleado técnicas de alta velocidad y corta amplitud, y como máximo se realizaron dos intentos por paciente (Martínez-Segura, Dela-Llave-Rincón, Ortega-Santiago, Cleland, y Fernández-de-Las-Peñas, 2012), y técnicas de baja velocidad y gran amplitud, durante 2 minutos (Calixtre et al., 2018). Ambos tipos de técnicas tenían por objetivo devolver la movilidad de la articulación en cuestión y se realizaron de forma bilateral (Dunning et al., 2016). 
Grupo 2 control $(G C)$. Se aplicó un tratamiento con un ligero contacto en la zona occipital sin intención de efecto durante 10 minutos (La-Touche et al., 2013), y a continuación, el paciente mantuvo una posición de reposo, sin ningún contacto físico, durante 10 minutos más (Espí-López et al., 2014).

\section{Evaluación}

En primer lugar, se recogieron las variables socio-demográficas así como las características de la migraña y se comprobó que se cumplieran los criterios de inclusión y exclusión. A continuación, los sujetos cumplimentaron el cuestionario Headache Impact Test (HIT-6) que evalúa el impacto de las cefaleas.

Evaluación clínica. Los datos recogidos fueron nombre completo, teléfono de contacto, sexo, edad, peso y altura. La información sobre las características de la migraña estaba en relación con la historia familiar de $\mathrm{M}$, edad de inicio, toma de medicación, localización de dolor, frecuencia de aparición e intensidad del dolor mediante la Escala Visual Analógica (EVA).

La EVA consiste en una línea de $10 \mathrm{~cm}$, dividida con números de 0 a 10 , siendo 0 ausencia de dolor y 10 peor dolor imaginable. Es un instrumento de medida de la intensidad del dolor válido y fiable, con un ICC de 0.97 (95\% CI=0.86 a 0.98) (Bijur, Silver, y Gallagher, 2001).

Cuestionario de Impacto de las Cefaleas (HIT-6). Publicado por Ware et al. (2000) para medir el impacto del dolor de cabeza. La puntuación resulta de la suma de: nunca (6 puntos), pocas veces ( 8 puntos), a veces (10 puntos), muy a menudo (11 puntos) y siempre (13 puntos). Con un total de 49 o menos puntos se considera "poco o ningún impacto", entre 50-55 “cierto impacto", entre 56-59 “impacto importante” y 60 o más "impacto muy severo". La consistencia interna es 0.89 ( $\alpha$ de Cronbach) y la fiabilidad de test-retest entre 0.78 y 0.90 (CCI) (Kosinski et al., 2003). Rendas et al. (2014) defienden que el HIT-6 es una herramienta fiable y válida evaluar el impacto de la M.

Pruebas de insuficiencia vertebrobasilar premanipulativa. La extensión y rotación de la cabeza y cuello del paciente es una prueba para descartar el riesgo de daño de la arteria vertebral (Swait y Finch, 2017). Será positivo si aparece nistagmo, náuseas, mareos o pérdida de conciencia (Côté, Kreitz, Cassidy, y Thiel, 1996)

\section{Análisis estadístico}

Los datos fueron analizados mediante el paquete estadístico SPSS v.24. Por un lado se realizó un análisis descriptivo, y por otro lado, una prueba ANOVA de medidas repetidas. El valor de significación que se estableció es $p<0.05$. 


\section{RESULTADOS}

De los 50 sujetos reclutados inicialmente, 44 cumplieron los criterios de inclusión. Tras la aleatorización, los sujetos fueron distribuidos en el GE $(n=22)$ y en el GC $(n=22)$. Todos los sujetos estuvieron presentes en todas las fases del estudio (Figura 1). Ningún sujeto dio positivo en la evaluación de compromiso de la arteria ni mostró efectos adversos por el tratamiento.

Figura 1. Diagrama de flujo del progreso de las fases del ensayo clínico aleatorizado paralelo de dos grupos según Declaración CONSORT 2010

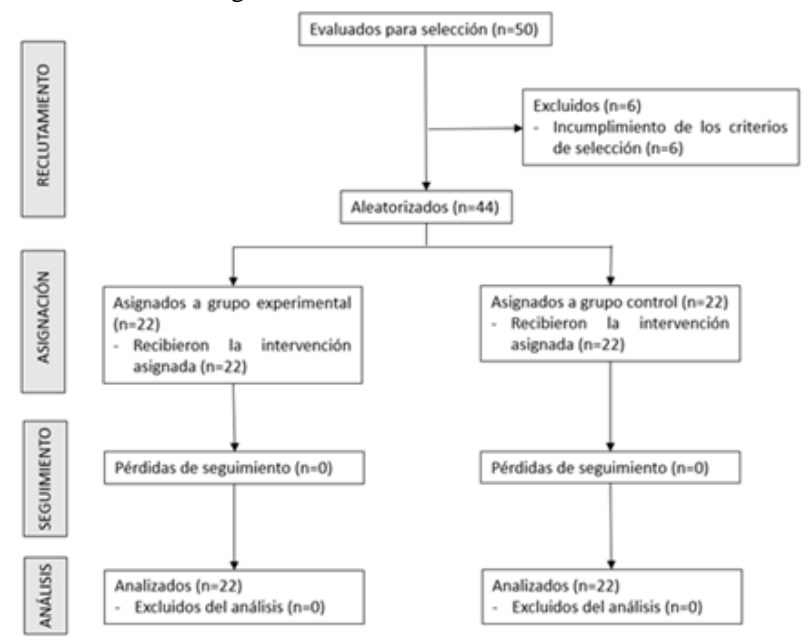

\section{Análisis descriptivos}

La muestra se formó por $36(81.80 \%)$ mujeres y 8 (18.20\%) hombres. Los resultados de los análisis descriptivos se similares en ambos grupos $(p>0.05)$ y se resumen en las tablas 1 y 2 .

Tabla 1. Frecuencias absolutas (frecuencias relativas) a las características demográficas y de la M

\begin{tabular}{llcc}
\hline \multirow{2}{*}{ Sexo } & & GE $(n=22)$ & GC $(n=22)$ \\
\hline \multirow{2}{*}{ Frecuencia de aparición } & Hombre & $4(18.2)$ & $4(18.2)$ \\
\cline { 2 - 4 } & Mujer & $18(91.8)$ & $18(81.8)$ \\
\cline { 2 - 4 } & \multicolumn{1}{c}{$>$ 15 días al mes } & $13(59.1)$ & $17(77.3)$ \\
\cline { 2 - 4 } Localización del dolor & Parieto-temporal (Sien) & $13(40.9)$ & $5(22.7)$ \\
\cline { 2 - 4 } & Zona orbitaria & $12(60.0)$ & $8(36.4)$ \\
\cline { 2 - 4 } & Frontal & $9(42.9)$ & $8(36.4)$ \\
\cline { 2 - 4 } & Occipital & $5(23.8)$ & $11(50.0)$ \\
\hline
\end{tabular}


Tabla 2. Media (Desviación estándar) de las características demográficas y de la M

\begin{tabular}{lcc}
\hline & $\mathrm{GE}(n=22)$ & $\mathrm{GC}(n=22)$ \\
\hline Edad (años) & $39.59(9.97)$ & $38.09(9.78)$ \\
\hline Edad de inicio (años) & $20.41(10.19)$ & $21.13(12.67)$ \\
\hline Tiempo de evolución (años) & $19.18(9.98)$ & $16.96(10.76)$ \\
\hline Duración (días) & $2.05(1.82)$ & $1.65(1.37)$ \\
\hline
\end{tabular}

\section{Análisis pre-intervención y post-intervención}

Se trata de un diseño experimental mixto. Se utilizó la prueba ANOVA de medidas repetidas. No se cumplió el supuesto de esfericidad mediante el test de esfericidad de Mauchly ( $p<0.05)$.

Efecto del tratamiento en la intensidad del dolor y el impacto de la M. No hubo diferencias significativas entre los dos factores (es decir, medidas tiempo y grupos) ni en HIT-6 ni en EVA ( $p>0.05$ ). La tabla 3 muestra los resultados obtenidos cuando el efecto del tratamiento fue analizado en cada grupo. Sin embargo, sí que hubo diferencias significativas en el grupo TMAV entre el pretratamiento y el postratamiento en EVA $(p=0.004)$ y en HIT-6 $(p=0.014)$.

Tabla 3. Efecto del tratamiento de la intensidad del dolor y del Test de Impacto de las Cefaleas

\begin{tabular}{lcccc}
\hline & & Pretratamiento & Postratamiento & $P$ valor \\
\hline \multirow{2}{*}{ EVA } & GC & $7.77(0.27)$ & $7.3(0.38)$ & 0.176 \\
\cline { 2 - 5 } & GE & $7.48(0.27)$ & $6.38(0.38)$ & $0.004^{*}$ \\
\hline \multirow{2}{*}{ HIT-6 } & GC & $63.5(1.34)$ & $61.5(1.69)$ & 0.227 \\
\cline { 2 - 5 } & GE & $65.32(1.34)$ & $61.14(1.69)$ & $0.014^{*}$ \\
\hline
\end{tabular}

\section{DISCUSIÓN Y CONCLUSIONES}

Desde nuestro conocimiento, este es el primer ensayo controlado aleatorizado que muestra que la TMAV sobre las regiones cervical, torácica y lumbosacra en pacientes con más de 4 episodios de migraña al mes mejora ciertos aspectos relacionados con la migraña, como la intensidad del dolor y el impacto asociado a esta.

De forma similar a estudios previos de TM en migraña, la mayoría de los participantes eran mujeres (Bevilaqua-Grossi et al., 2016; Chaibi, Benth, Tuchin, y Russell, 2017; Espí-López et al., 2018; Garrigós-Pedrón et al., 2018; Ghanbari, Askarzadeh, Petramfar, y Mohamadi, 2015; Voigt et al., 2011), con una percepción de dolor moderada-severa (Bevilaqua-Grossi et al., 2016; Espí-López et al., 2018; GarrigósPedrón et al., 2018; Ghanbari et al., 2015; Tuchin, Pollard, y Bonello, 2000; Voigt et al., 2011), y la localización del dolor era principalmente en la sien.

Se obtuvo una disminución de la intensidad del dolor de 1,1 puntos en el GE. Trabajos anteriores muestran que las técnicas de manipulación y articulatorias son efectivas en la reducción de la intensidad en la migraña (Bevilaqua-Grossi et al., 2016; Calixtre et al., 2018; Cerritelli et al., 2015; Chaibi et al., 2017; Garrigós-Pedrón et al., 
2018; Tuchin et al., 2000; Voigt et al., 2011). Por ejemplo, la aplicación de la TMAV según el método Gonstead, con contacto específico, velocidad alta, corta amplitud y directo sobre la disfunción biomecánica espinal según el diagnóstico por test quiroprácticos estándar, mostró una disminución significativa de 0.7 puntos al mes de la intervención y a los 3 meses, 1.3 puntos a los 6 meses y 0.6 puntos a los 12 meses, en pacientes con al menos 1 episodio de migraña al mes (Chaibi et al., 2017).

Estos hallazgos están apoyados por la revisión sistemática realizada por Rist et al. (2019) la cual afirma que la aplicación de la manipulación espinal puede ser efectiva en la reducción de los días con migraña, la intensidad del dolor y la discapacidad. Los resultados de nuestro estudio confirman que las técnicas manipulativas y articulatorias son efectivas en la reducción de la intensidad del dolor de la migraña y los efectos positivos pueden observarse a las 4 semanas.

En cuanto al impacto de la cefalea, el cuestionario HIT-6 del GE mostró una reducción significativa de 4.18 puntos. Otros autores también han medido esta variable en pacientes con migraña (Calixtre et al., 2018; Cerritelli et al., 2015; Espí-López et al., 2018; Garrigós-Pedrón et al., 2018). Cerritelli et al. (2015) realizaron un ensayo controlado aleatorizado con 105 sujetos divididos en tres grupos: a) un grupo de terapia manual osteopática que incluía técnicas manipulativas, craneales y viscerales, b) grupo placebo, con contacto inespecífico, y c) grupo control. Obtuvieron una disminución significativa en la puntuación HIT-6 en el primer grupo de 5,6 puntos a los 6 meses. Sin embargo, en comparación con el actual estudio, realizaron una combinación de técnicas tanto articulares y manipulativas como de tejido blando, y no fue uniforme en todos los pacientes. Por tanto, la TMAV sobre la zona cervicotorácica y lumbosacra es efectiva al reducir el impacto asociado a la migraña.

La evidencia sugiere que la terapia articulatoria vertebral puede estimular sistemas de inhibición neural al activar vías inhibitorias descendentes centrales (Chaibi et al., 2017), así como alterar las tasas de descarga aferentes al estimular mecanorreceptores y propioceptores, cambiando los niveles de excitabilidad de las motoneuronas alfa y la consiguiente actividad muscular (Dunning et al., 2016). Estos mecanismos son interesantes emplearlos en pacientes con migraña no solo a nivel cervicotorácico si no también lumbosacro, para tratar al paciente de forma global y obtener resultados más positivos (Cerritelli et al., 2015; Voigt et al., 2011). Aunque en el presente estudio no se observaron efectos adversos, es importante tener en cuenta la posibilidad de disección de la arteria vertebral durante la manipulación para determinar los cuidados más apropiados a cada paciente (Aleksander, Benth, Tuchin, y Russell, 2017).

Una de las limitaciones del presente estudio es la falta de un seguimiento a largo plazo y debería considerarse en futuros estudios. Por otra parte, la muestra constaba de sujetos con migraña tanto episódica como crónica, y en el futuro convendría 
analizar los resultados por separado para observar si responden de forma diferente al tratamiento.

En conclusión, la aplicación de TMAV en pacientes con más de 4 episodios de migraña al mes, disminuye la intensidad del dolor y el impacto asociado a la migraña después de 4 sesiones, y podría ser un tratamiento adecuado para combinar con la medicación.

Agradecimientos a la Dra. Gemma Victoria Espí López.

\section{REFERENCIAS}

Abu Bakar, N., Tanprawate, S., Lambru, G., Torkamani, M., Jahanshahi, M., y Matharu, M. (2016). Quality of life in primary headache disorders: A review. Cephalalgia: An International Journal of Headache, 36(1), 67-91. doi: 10.1177/0333102415580099

Bevilaqua-Grossi, D., Gonçalves, M.C., Carvalho, G.F., Florencio, L.L., Dach, F., Speciali, J.G.,... Chaves, T.C. (2016). Additional Effects of a Physical Therapy Protocol on Headache Frequency, Pressure Pain Threshold, and Improvement Perception in Patients with Migraine and Associated Neck Pain: A Randomized Controlled Trial. Archives of Physical Medicine and Rehabilitation, 97(6), 866-874. doi:10.1016/j.apmr.2015.12.006

Bijur, P.E., Silver, W., y Gallagher, E.J. (2001). Reliability of the visual analog scale for measurement of acute pain. Academic Emergency Medicine: Official Journal of the Society for Academic Emergency Medicine, 8(12), 1153-1157.

Calixtre, L.B., Oliveira, A.B., de Sena Rosa, L.R., Armijo-Olivo, S., Visscher, C.M., y Alburquerque-Sendín, F. (2018). Effectiveness of mobilisation of the upper cervical region and craniocervical flexor training on orofacial pain, mandibular function and headache in women with TMD. A randomised, controlled trial. Journal of Oral Rehabilitation, 46(2), 109-119. doi: 10.1111/joor.12733.

Cerritelli, F., Ginevri, L., Messi, G., Caprari, E., Di Vincenzo, M., Renzetti, C., ... Provinciali, L. (2015). Clinical effectiveness of osteopathic treatment in chronic migraine: 3-Armed randomized controlled trial. Complementary Therapies in Medicine, 23(2), 149-156. doi: 10.1016/j.ctim.2015.01.011.

Chaibi, A., Benth, J.Š., Tuchin, P.J., y Russell, M.B. (2017). Adverse events in a chiropractic spinal manipulative therapy single-blinded, placebo, randomized controlled trial for migraineurs. Musculoskeletal Science y Practice, 29, 66-71. doi:10.1016/j.msksp.2017.03.003

Chaibi, A., Benth, J.S., Tuchin, P.J., y Russell, M.B. (2017). Chiropractic spinal manipulative therapy for migraine: a three-armed, single-blinded, placebo, randomized controlled trial. European Journal of Neurology, 24(1), 143-153. doi:10.1111/ene.13166

Chaibi, A., y Russell, M.B. (2019). A risk-benefit assessment strategy to exclude cervical artery dissection in spinal manual-therapy: a comprehensive review. Annals of Medicine, 51(2), 118-127. doi:10.1080/07853890.2019.1590627.

Charles, A. (2018). The pathophysiology of migraine: implications for clinical management. The Lancet. Neurology, 17(2), 174-182. doi: 10.1016/S1474-4422(17)30435-0.

Côté, P., Kreitz, B., Cassidy, J., y Thiel, H. (1996). The validity of the extension-rotation test as a clinical screening procedure before neck manipulation: a secondary analysis. Journal of Manipulative and Physiological Therapeutics, 19(3), 159-64. 
Dunning, J.R., Butts, R., Mourad, F., Young, I., Fernandez-de-Las Peñas, C., Hagins, M., ... Cleland, J.A. (2016). Upper cervical and upper thoracic manipulation versus mobilization and exercise in patients with cervicogenic headache: a multi-center randomized clinical trial. BMC Musculoskeletal Disorders, 17, 64. doi:10.1186/s12891016-0912-3

Espí-López, G., Gómez-Conesa, A., Gómez, A., Martínez, J., Pascual-Vaca, A., y Blanco, C. (2014). Treatment of tension-type headache with articulatory and suboccipital soft tissue therapy: A double-blind, randomized, placebo-controlled clinical trial. Journal of Bodywork and Movement Therapies, 18(4), 576-85. doi:10.1016/j.jbmt.2014.01.001

Espí-López, G.-V., Ruescas-Nicolau, M.-A., Nova-Redondo, C., Benítez-Martínez, J. C., Dugailly, P.-M., y Falla, D. (2018). Effect of Soft Tissue Techniques on Headache Impact, Disability, and Quality of Life in Migraine Sufferers: A Pilot Study. Journal of Alternative and Complementary Medicine, 24(11), 1099-1107. doi:10.1089/acm.2018.0048

Fernández-de-Las-Peñas, C., y Cuadrado, M.L. (2016). Physical therapy for headaches. Cephalalgia: An International Journal of Headache, 36(12), 1134-1142. doi:10.1177/0333102415596445

Ferracini, G.N., Florencio, L.L., Dach, F., Bevilaqua, D., Palacios-Ceña, M., Ordás-Bandera, C., ... Fernández-de-Las-Peñas, C. (2017). Musculoskeletal disorders of the upper cervical spine in women with episodic or chronic migraine. European Journal of Physical and Rehabilitation Medicine, 53(3), 342-350. doi:10.23736/S1973-9087.17.04393-3

Garrigós-Pedrón, M., La Touche, R., Navarro-Desentre, P., Gracia-Naya, M., y Segura-Ortí, E. (2018). Effects of a Physical Therapy Protocol in Patients with Chronic Migraine and Temporomandibular Disorders: A Randomized, Single-Blinded, Clinical Trial. Journal of Oral y Facial Pain and Headache, 32(2), 137-150.

Ghanbari, A., Askarzadeh, S., Petramfar, P., y Mohamadi, M. (2015). Migraine responds better to a combination of medical therapy and trigger point management than routine medical therapy alone. NeuroRehabilitation, 37(1), 157-163. doi:10.3233/NRE-151248

Goadsby, P.J., Holland, P.R., Martins-Oliveira, M., Hoffmann, J., Schankin, C., y Akerman, S. (2017). Pathophysiology of Migraine: A Disorder of Sensory Processing. Physiological Reviews, 97(2), 553-622. doi:10.1152/physrev.00034.2015

Headache Classification Committee of the International Headache Society (IHS) (2013). The International Classification of Headache Disorders, 3rd edition (beta version). Cephalalgia, 33, 629-808.

Kosinski, M., Bayliss, M., Bjoner, J., Ware, J., Garber, W., Batenhorst, A., et al. (2003). A sixitem short-form survey for measuring headache impact: the HIT-6. Quality of Life Research, 12(8), 963-74. doi:10.1023/A:1026119331193

La-Touche, R., París-Alemany, A., Mannheimer, J., Angulo-Díaz-Parreño, S., Bishop, M., LópezValverde-Centeno, A., y et al. (2013). Does mobilization of the upper cervical spine affect pain sensitivity and autonomic nervous system function in patients with cervicocraniofacial pain?: A randomized-controlled trial. The Clinical Journal of Pain, 29, 20515. doi:10.1097/AJP.0b013e318250f3cd

Luedtke, K., Starke, W., y May, A. (2018). Musculoskeletal dysfuction in migraine patients. Cephalalgia, 38(5), 865-875. doi:10.1177/0333102417716934 
Martínez-Segura, R., De-la-Llave-Rincón, A.I., Ortega-Santiago, R., Cleland, J.A., y Fernándezde-Las-Peñas, C. (2012). Immediate changes in widespread pressure pain sensitivity, neck pain, and cervical range of motion after cervical or thoracic thrust manipulation in patients with bilateral chronic mechanical neck pain: a randomized clinical trial. The Journal of Orthopaedic and Sports Physical Therapy, 42(9), 806-814. doi:10.2519/jospt.2012.4151

Moore, C.S., Sibbritt, D.W., y Adams, J. (2017). A critical review of manual therapy use for headache disorders: prevalence, profiles, motivations, communication and self-reported effectiveness. BMC Neurology, 17(1), 61. doi:10.1186/s12883-017-0835-0

OMS (2019). Cefaleas. Recuperado de WHO Media centre website: http://www.who.int/mediacentre/factsheets/fs277/es/

Rendas, R., Yang, M., Varon, S., DeGryse, R., y Kosinski, M. (2014). Validation of the Headache Impact Test (HIT-6) in patients with chronic migraine. Health and Quality of Life Outcomes, 12, 117. doi: 10.1186/s12955-014-0117-0.

Rist, P.M., Hernandez, A., Bernstein, C., Kowalski, M., Osypiuk, K., Vining, R.,... Wayne, P.M. (2019). The Impact of Spinal Manipulation on Migraine Pain and Disability: A Systematic Review and Meta-Analysis. Headache, 59(4), 532-542. doi:10.1111/head.13501

Steiner, T., Stovner, L., y Vos, T. (2016). Global Burden Disease 2015: migraine is the third cause of disability in under 50s. The Journal of Headache and Pain, 17, 104. doi:10.1186/s10194-016-0699-5

Swait, G., y Finch, R. (2017). What are the risks of manual treatment of the spine? A scoping review for clinicians. Chiropractic \& Manual Therapies, 25(1), 37. doi:10.1186/s12998017-0168-5

Tuchin, P.J., Pollard, H., y Bonello, R. (2000). A randomized controlled trial of chiropractic spinal manipulative therapy for migraine. Journal of Manipulative and Physiological Therapeutics, 23(2), 91-95.

Voigt, K., Liebnitzky, J., Burmeister, U., Sihvonen-Riemenschneider, H., Beck, M., Voigt, R., y Bergmann, A. (2011). Efficacy of osteopathic manipulative treatment of female patients with migraine: results of a randomized controlled trial. Journal of Alternative and Complementary Medicine, 17(3), 225-230. doi:10.1089/acm.2009.0673

Ware, J., Bjorner, J., y Kosinski, M. (2000). Practical implications of item response theory (IRT) and computer adaptive testing. Medical Care, 38(2), 73-83.

Recibido: 8 de julio de 2019

Recepción Modificaciones: 30 de julio de 2019

Aceptado: 12 de septiembre de 2019 